\title{
Tumor-mesenchymal stem cell interactions: therapeutic implications for epithelial ovarian cancer
}

\author{
Alain Piché \\ Département de Microbiologie et Infectiologie, Faculté de Médecine, Université de Sherbrooke, QC, Canada \\ Correspondence to: Alain Piché. Département de Microbiologie et Infectiologie, Faculté de Médecine, Université de Sherbrooke, QC J1H 5N4, \\ Canada. Email: Alain.piche@usherbrooke.ca. \\ Comment on: Pasquier J, Gosset M, Geyl C, et al. CCL2/CCL5 secreted by the stroma induce IL-6/PYK2 dependent chemoresistance in ovarian \\ cancer. Mol Cancer 2018;17:47.
}

Submitted May 15, 2018. Accepted for publication May 17, 2018.

doi: $10.21037 /$ tcr.2018.05.41

View this article at: http://dx.doi.org/10.21037/tcr.2018.05.41

Epithelial ovarian cancer (EOC) is the most common cause of gynecological cancer-related death (1). When compared to most solid tumors, EOC has a distinct mechanism of dissemination. Cells detach from the primary tumor and aggregate in peritoneal fluid to form free-floating multicellular spheroids, which then travel through the peritoneal fluid at distant sites onto the mesothelial lining where metastatic outgrowth occurs (2). Because this process is clinically silent, most women $(\sim 70 \%)$ present with metastasis throughout the peritoneal cavity. The current standard of care for EOC is debulking surgery combined with platinum-based chemotherapy (3). Drug resistance is one of the major obstacles in the treatment of EOC. EOC resistance can arise within tumor cells as a consequence of genetic changes leading to either intrinsic or acquired resistance (4). In addition, soluble factors and stromal cells within the tumor environment, which can promote the inhibition of drug-induced apoptosis, may constitute another mechanism of drug resistance (4). Despite optimal tumor-debulking surgery and initial high responses rates to first-line chemotherapy, most patients with advanced EOC relapse within $18-22$ months with a disease that is still sensitive to the first-line therapy suggesting that their state of chemoresistance was transient and not associated with genetic changes in tumor cells in contrast to acquired or intrinsic resistance. This transient drug resistance state is thought to be driven by the surrounding tumor environment in EOC.

A feature that distinguishes EOC from other solid tumors is the unique tumor environment constituted by ascites. Ascites accumulates during EOC progression. Ascites comprises detached tumor and activated mesothelial cells, multicellular spheroids, adaptive immune cells, and a number of other host cells. In addition, cell-free ascites constitutes a proinflammatory reservoir of cytokines, chemokines, growth factors, and extracellular matrix (ECM) fragments $(5,6)$. All together, these soluble factors contribute to create a pro-survival environment that lead to resistance and relapse by preventing drug-induced apoptosis $(7,8)$. Indeed, a number of ligands found in ascites, including hepatocyte growth factor (HGF) (9-11), lysophosphatidic acid (LPA) (12) and CCL18 (13) have been shown to promote EOC cell growth and survival. Although dissecting the role of different individual factors from ascites remains an important goal in the field, the overall pro-survival effect of ascites is likely to result from the combined actions of soluble factors and tumor-host cell interactions. If we are to improve the overall survival of EOC patients, it will be important to gain a better understanding of the mechanisms underlying the transient state of resistance induce by the surrounding environment.

Among the different stromal cells present in ascites, cancer associated-mesenchymal stem cells (MSCs) are pluripotent cells that have the capacity to differentiate into various cell types such as osteoclasts, myofibroblasts, chondrocytes, adipocytes, and cancer-associated fibroblast (CAFs). MSCs are important components of the tumor environment. These cells are characterized by the expression of CD90, CD105, CD29, CD73 and the lack of CD45, CD34, CD11b, CD19 and HLA-DR (14). Cancer 
associated-MSCs are central in establishing a pre-metastatic niche and promoting metastasis. MSCs exert their growth enhancing effects by paracrine secretion of growth and anti-apoptotic factors (15-17). There are numerous reports in which co-culturing MSCs with tumor cells inhibited drug-induced apoptosis in haematological malignancies or solid cancers (18-20). There is also evidence to suggest that the properties of these cells are strongly influenced by both surrounding soluble factors and the tumor microenvironment.

Paracrine signaling through MSCs can confer resistance to chemotherapy in EOC cells (21). In a recent article, Pasquier and colleagues highlight the role of MSCs in promoting EOC cells resistance to chemotherapy (22). First, the authors isolated from stage III serous EOC ascites cells that stained positive for CD73, CD105, CD29 and CD90 but negative for CD45 and Lin. In addition, these cells displayed an adherent fibroblastic morphology in culture, defining them as MSCs. Using a transwell setting of MSCs and EOC cell co-culture, they showed that factor secreted by MSCs inhibited carboplatin and taxol-induced apoptosis. Tumor cell survival was further increased by preexposing EOC cells to MSCs supernatants for $48 \mathrm{~h}$ prior to adding drugs. Interestingly, IL-6 or IL-6-R blocking antibodies completely abrogated MSC supernatantmediated inhibition of drug-induced apoptosis suggesting that IL-6 signaling is crucial in this process. Using an intraperitoneal (i.p.) xenograft mouse model, Pasquier and colleagues showed that the presence of MSCs within the tumor nodules enhanced the resistance to chemotherapy. Conversely, i.p. injection of tocilizumab, a humanized antiIL-6-R blocking antibody, completely abrogated MSCsmediated pro-survival effect in vivo, further supporting the role of IL-6 in chemoresistance induced by cancerassociated MSCs.

The crucial role of IL-6 in tumor-host cell signaling is consistent with previous studies showing high levels of IL-6 and soluble IL-6-R in EOC ascites (23) as well as with the fact that IL-6 levels increased over the course of EOC progression in ascites (24). In previous studies, mesothelial and tumor cells have been identified as important sources of IL-6 in ascites (25). In their study, Pasquier and colleagues determine that IL- 6 was produced by the tumor cells in response to exposure to MSC supernatant. Indeed, shRNAmediated downregulation of IL-6 in EOC cells abrogated the protecting effect of MSCs against chemotherapy. To identify which factor(s) secreted by MSCs could induce the secretion of IL- 6 by tumor cells, the authors used a cytokine array to analyze the MSC supernatant content. They showed that CCL2 and CCL5 were the most potent chemokines in MSC supernatant to induce IL-6 secretion. Phosphorylation kinase profiling revealed that the prolinerich tyrosine kinase 2 (PYK2) was particularly increased in tumor cells in response to MSC supernatant and its phosphorylation was inhibited by tocilizumab and IL-6 shRNA suggesting that PYK2 phosphorylation was IL-6dependent.

Taken together, these results suggest MSCs could play a significant role in the transient drug resistance state associated with the surrounding tumor environment. Being intrinsically resistant to chemotherapy (as shown in this study), MSCs will survive cytotoxic treatments and will drive EOC recurrence by enabling tumor cells to survive treatments. This study highlights not only the importance but also the complexity of tumor-host cell interactions that can lead to drug resistance. As suggested by the authors, this work emphasizes the potential of targeting the tumor environment as a novel mean to improve the overall survival of patients with EOC.

Although the study by Pasquier and colleagues is an important contribution to the field, there are a number of question that remain to be addressed. For example, floating multicellular spheroids in peritoneal fluid are mostly likely pivotal in mediating the state of transient resistance. Whether these spheroids contain MSCs that could signal adjacent tumor cells to promote drug resistance remains to be determined. It would also be interesting to determine whether the levels of IL-6 in ascites correlates with the emergence of resistance in EOC patients. Furthermore, since IL-6 concentrations used to demonstrate enhanced drug resistance in vitro were about a 100 -fold relative to the concentration of IL-6 secreted by EOC cell lines upon stimulation with CCL2 or CCL5, it would have been interesting if Pasquier and colleagues would have performed an IL-6 dose-response curves. Given its potential role in tumor cell resistance to drug-induced apoptosis, it will be interesting to assess IL-6 as a predictive marker of drug resistance.

Despite these limitations, the role of cancer-associated MSCs in drug resistance remains an interesting finding which support previous results by this group. However, the mechanisms underlying the transient drug-resistant state mediated by ascites is probably far more complex than just the contribution of MSCs cells and is likely the result from the integration of the various cytokines, chemokines and growth factors cell signaling in addition to the multiple cell- 
cell crosstalks that occurs within this particular peritoneal fluid.

\section{Acknowledgments}

Funding: None.

\section{Footnote}

Provenance and Peer Review: This article was commissioned and reviewed by the Section Editor Da Li (Department of Obstetrics and Gynecology, Shengjing Hospital of China Medical University, Shenyang, China).

Conflicts of Interest: The author has completed the ICMJE uniform disclosure form (available at http://dx.doi. org/10.21037/tcr.2018.05.41). The author has no conflicts of interest to declare.

Ethical Statement: The author is accountable for all aspects of the work in ensuring that questions related to the accuracy or integrity of any part of the work are appropriately investigated and resolved.

Open Access Statement: This is an Open Access article distributed in accordance with the Creative Commons Attribution-NonCommercial-NoDerivs 4.0 International License (CC BY-NC-ND 4.0), which permits the noncommercial replication and distribution of the article with the strict proviso that no changes or edits are made and the original work is properly cited (including links to both the formal publication through the relevant DOI and the license). See: https://creativecommons.org/licenses/by-nc-nd/4.0/.

\section{References}

1. Siegel RL, Miller KD, Jemal A. Cancer statistics, 2017. CA Cancer J Clin 2017;67:7-30.

2. Shield K, Ackland ML, Ahmed N, et al. Multicellular spheroids in ovarian cancer metastases: biology and pathology. Gynecol Oncol 2009;113:143-8.

3. Bast RC, Hennessy B, Mills GB. The biology of ovarian cancer: new opportunities for translation. Nat Rev Cancer 2009;9:415-28.

4. Pogge von Strandmann E, Reinartz S, Wager U, et al. Tumor-host cell interactions in ovarian cancer: pathways to therapy failure. Trends in Cancer 2017;3:137-48.

5. Matte I, Lane D, Laplante C, et al. Profiling of cytokines in human epithelial ovarian cancer ascites. Am J Cancer Res 2012;2:566-80.

6. Giuntoli RL, Webb TJ, Zoso A, et al. Ovarian cancerassociated ascites demonstrates altered immune environment: implications for antitumor immunity. Anticancer Res 2009;29:2875-84.

7. Lane D, Goncharenko-Khaider N, Rancourt C, et al. Ovarian cancer ascites protects from TRAIL-induced cell death through $\alpha v \beta 5$ integrin-mediated focal adhesion kinase and Akt activation. Oncogene 2010;29:3519-31.

8. Goncharenko-Khaider N, Matte I, Lane D, et al. Ovarian cancer ascites increase Mcl-1 expression in tumor cells through ERK1/2-Elk-1 signaling to attenuate TRAILinduced apoptosis. Mol Cancer 2012;11:84.

9. Nakamura M, Ono YJ, Kanemura M, et al. Hepatocyte growth factor secreted by ovarian cancer cells stimulates peritoneal implantation via the mesothelial-mesenchymal transition of the peritoneum. Gynecol Oncol 2015;139:345-54.

10. Matte I, Lane D, Laplante C, et al. Ovarian cancer ascites enhance the migration of patient-derived peritoneal mesothelial cells via cMet pathway through HGFdependent and -independent mechanisms. Int J Cancer 2015;137:289-98.

11. Kwon Y, Smith BD, Zhou Y, et al. Effective inhibition of c-Met-mediated signaling, growth and migration of ovarian cancer cells is influenced by the ovarian tissue microenvironment. Oncogene 2015;34:144-53.

12. Mills GB, Moolenar WH. The emerging role of lysophosphatidic acid in cancer. Nat Rev Cancer 2003;3:582-91.

13. Lane D, Matte I, Laplante C, et al. CCL18 from ascites promotes ovarian cancer cell migration through prolinerich tyrosine kinase 2 signaling. Mol Cancer 2016;15:58.

14. Reagan MR, Kaplan DR. Concise review: Mesenchymal stem cell-tumor homing: detection methods in disease model systems. Stem Cells 2011;29:920-7.

15. Hung SC, Pochampally RR, Chen SC, et al. Angiogenic effects of human multipotent stromal cell conditioned medium activate the PI3K-Akt pathway in hypoxic endothelial cells to inhibit apoptosis, increase survival, and stimulate angiogenesis. Stem Cells 2007;25:2363-70.

16. Mishra PJ, Humeniuk R, Medina DJ, et al. Carcinomaassociated fibroblast-like differentiation of human mesenchymal stem cells. Cancer Res 2008;68:4331-9.

17. Dawson MR, Chae SS, Jain RK, et al. Direct evidence for lineage-dependent effects of bone marrow stromal cells on tumor progression. Am J Cancer Res 2011;1:144-54. 
18. Vianello F, Villanova F, Tisato V, et al. Bone marrow mesenchymal stromal cells non-selectively protect chronic myeloid leukemia cells from imatinib-induced apoptosis via the CXCR4\CXCL12 axis. Haematologica 2010;95:1081-9.

19. Balakrishnan K, Burger JA, Quiroga MP, et al. Influence of bone marrow stromal microenvironment on forodesineinduced responses in CLL primary cells. Blood 2010;116:1083-91.

20. Scherzed A, Hackenberg S, Froelich K, et al. BMSC enhance the survival of paclitaxel treated squamous cell carcinoma in vitro. Cancer Biol Ther 2011;11:349-57.

21. Lis R, Touboul C, Mirshahi P, et al. Tumor associatedmesenchymal stem cells protects ovarian cancer cells from hyperthermia through CXCL12. Int J Cancer 2011;128:715-25.

Cite this article as: Piché A. Tumor-mesenchymal stem cell interactions: therapeutic implications for epithelial ovarian cancer. Transl Cancer Res 2018;7(Suppl 7):S711-S714. doi: 10.21037/tcr.2018.05.41
22. Pasquier J, Gosset M, Geyl C, et al. CCL2/CCL5 secreted by the stroma induce IL-6/PYK2 dependent chemoresistance in ovarian cancer. Mol Cancer 2018;17:47.

23. Lane D, Matte I, Rancourt C, et al. Prognostic significance of IL-6 and IL-8 ascites levels in ovarian cancer patients. BMC Cancer 2011;11:210.

24. Matte I, Bessette PH, Piché A. Ascites in ovarian cancer progression: opportunities for biomarker discovery and new avenues for targeted therapies. INTECH open access publisher, Chapter 9 in Ascites - pathophysiology, treatment, complication and prognosis, 2017.

25. Offner FA, Obrist P, Stadlmann S, et al. IL-6 secretion by human peritoneal mesothelial and ovarian cancer cells. Cytokine 1995;7:542-7. 